\title{
Lineamientos de ordenamiento y desarrollo territorial para Sincelejo desde el modelo de territorios inteligentes
}

Territorial planning and development guidelines for Sincelejo from the smart territories model

\section{Diretrizes de planejamento e desenvolvimento territorial para Sincelejo a partir do modelo de territórios inteligentes}

Jenny Milena Buelvas Salgado

Arquitecta, MSc. Urbanismo y Desarrollo Territorial

Corporación Universitaria del Caribe-CECAR, Sincelejo.

jbuelvas07@hotmail.com

(iD) https://orcid.org/0000-0002-4198-8073

Recibido: octubre 9 de 2021

Aceptado: diciembre 10 de 2021

Publicado: diciembre 15 de 2021

\section{RESUMEN}

La ciudad de Sincelejo, Colombia, al estar catalogada de ciudad emergente con una población intermedia, se plantea que podría proyectar su desarrollo económico, social y físico-construido a partir de los modelos estratégicos que definen a los territorios inteligentes, por lo que como objetivo principal de esta Investigación- se pretende identificar los Lineamientos de Ordenamiento y Desarrollo Territorial para Sincelejo desde el Modelo Conceptual Territorio Inteligente, según una metodología estratégica. Los resultados develan un estado del arte que lleva a identificar brechas criticas para los subsistemas del modelo y, en consecuencia, a proponer mejoras para Sincelejo.

Palabras clave: Territorio Inteligente; Sincelejo; desarrollo territorial; ordenación.

\section{ABSTRACT}

Since the city of Sincelejo, Colombia, is classified as an emerging city with an intermediate population, it is proposed that it could project its economic, social and physical-built development based on the strategic models that define smart territories, therefore -as the main objective of this research - the aim is to identify the Territorial Planning and Development Guidelines for Sincelejo from the Smart Territory Conceptual Model, according to a strategic methodology. The results reveal a state of the art that leads to identifying critical gaps for the subsystems of the model and, consequently, to propose improvements for Sincelejo.

Keywords: Smart territory; Sincelejo; territorial development; ordination. 


\section{RESUMO}

A cidade de Sincelejo, Colômbia, por ser classificada como uma cidade emergente com uma população intermediária, propõe-se que possa projetar seu desenvolvimento econômico, social e físico construído a partir dos modelos estratégicos que definem territórios inteligentes, portanto -como o principal objetivo deste pesquisa- pretende-se identificar as Directrizes de Planeamento e Desenvolvimento Territorial para o Sincelejo a partir do modelo conceptual de território inteligente, segundo uma metodologia estratégica. Os resultados revelam um estado da arte que leva a identificar lacunas críticas para os subsistemas do modelo e, consequentemente, propor melhorias para o Sincelejo.

Palavras-chave: Território inteligente; Sincelejo; desenvolvimento territorial; ordenação

\section{INTRODUCCIÓN}

La ciudad de Sincelejo, catalogada como 'La Perla de la Sabana', es la capital del Departamento de Sucre, centro subregional del sistema urbano del Caribe colombiano. De acuerdo con el Departamento Nacional de Planeación - DNP, Sincelejo, a 2016, alcanzó una población de 270.031 habitantes de la población total de 22.458.559 que habitan en las ciudades capitales, albergando así el $1.2 \%$ del total de la población que vive en las ciudades de Colombia.

Sincelejo es un territorio que despierta el interés investigativo por las implicaciones que supone hablar de desarrollo territorial debido a las características de la estructura productiva del departamento de Sucre. Esta ciudad posee una alta dependencia del sector, un incipiente desarrollo en la industria y servicios, y una débil integración a la economía nacional e internacional, situándose en el espacio como un territorio de ordenación fija, a lo que contribuye el hecho de que se caracteriza como ciudad autónoma, es decir, que su actividad económica, capacidad institucional y de gestión, así como su crecimiento poblacional, se presenta principalmente dentro de sus límites político-administrativos, y su interdependencia con otras ciudades vecinas aún no es tan fuerte, por lo que califica como ciudad autónoma y uni-nodal. Por otra parte, a la histórica alta tasa de crecimiento demográfico se suman algunas características de los productos de expansión, principalmente la ganadería, con bajo encadenamiento y alta dependencia de la demanda doméstica, que imponen restricciones al crecimiento económico regional a largo plazo.
Esta última conjunción de factores, junto a la distribución de la tierra, ayudan a explicar la evolución de la economía sucreña desde sus inicios, como Departamento de reciente creación en 1967. Como lo anota DANE (2014,) el Departamento de Sucre históricamente ha contribuido con el $0,8 \%$ del Producto Interno Bruto (PIB) del país, cifra que poco ha variado desde 1981 hasta 2013.

La situación descrita para Sincelejo es plausible de cambio desde las renovada teoría sobre el Smart Place o Territorio Inteligente (Vergara, 2009), en referencia a la importancia que adquiere la ciudad frente a la región en el marco de la economía espacial, desde la cual se vislumbra este municipio como parte del "Diamante Caribe y Santanderes de Colombia" iniciativa impulsada por el Gobierno Nacional, la cual, contempla el fortalecimiento del Sistema de Ciudades como motor del crecimiento del país, para lo cual se propone en la "Visión Colombia 2019: Construir Ciudades Amables y la Política Nacional para Consolidar el Sistema de Ciudades de Colombia a 2035." (Plan Nacional de Desarrollo, 2014 - 2018)

¿Pero por qué a Sincelejo, un municipio en condiciones de pobreza histórica, que desmejora aún más para el resto de Municipios de Sucre, se está ahora reconociendo con las potencialidades que se le endilgan a la economía espacial de los lugares centrales?

Parte de la respuesta a este interrogante se encuentra precisamente en su ubicación, misma de la que se ocupa la teoría de los 
lugares centrales (TLC), la cual defiende que "un sistema territorial sustentable y eficiente conduce a mayores niveles de crecimiento, el cual, dependiendo del grado de democracia real, conduce al desarrollo, y mayores niveles de desarrollo potencian la sinergia, dando como resultado un proceso de causación circular acumulativa de tipo virtuoso" (Santacruz, 2006, p. 158).

Son entre otros autores Weber (1929) y Christaller (1966), quienes contribuyen al desarrollo de la Teoría de los Lugares Centrales, al determinar los elementos más importantes de la economía espacial en razón de las distancias, la localización, el comportamiento de la actividad económica en el espacio; pero, de manera más específica, Lösch (1944) en su trabajo "Die räumliche ordnung der wirtschaft", plantea que alrededor de un lugar central se formará un pequeño núcleo en el que cada área comercial es tangente a otra seis. Asimismo, los sistemas urbanos se conforman con base en la jerarquía de los lugares centrales, como lugares de oferta de bienes centrales y como puntos intermedios de redes de transporte. (Santacruz, 2006, p. 144).

Llegados a este punto, es necesario mirar a Sincelejo bajo la lupa de la TLC, tal como es descrita en la iniciativa Diamante Caribe:

Sincelejo se localiza en un punto privilegiado para las comunicaciones en el que confluyen la Troncal 25, que conecta con Montería y con Medellín a través de Caucasia, y la Carretera 90, Transversal del Caribe, que comunica con Cartagena y Barranquilla. Sincelejo es punto obligado de paso en las conexiones desde la costa del Golfo de Morrosquillo hacia el interior. A través de la Carretera 25, Sincelejo tiene acceso a El Carmen de Bolívar y de ahí al paso sobre el Magdalena en Plato y al puerto fluvial de Magangué. Estas conexiones colocan a la ciudad en una posición estratégica como centro de acopio y distribución logística, y como centro de producción de bienes de gran consumo. Su ámbito de influencia en una hora de viaje se limita a las zonas del Centro y Norte del departamento de Sucre; así como algunas áreas del Oeste de Bolívar, mientras que el Sur de Sucre permanece desconectado debido a la falta de carreteras que conecten con la zona de La Mojana y la zona del entorno del río San Jorge. En la isócrona de dos horas, donde todavía la influencia funcional de la ciudad se deja sentir, aparecen áreas de influencia cruzada con Montería y se alcanza el río Magdalena en Magangué y el Canal del Dique en Arjona y Calamar, ya en el ámbito funcional de Cartagena. Es especialmente destacable que, para distancias mayores, Sincelejo tiene una localización de mucha mayor centralidad que cualquier otra ciudad del Diamante. En un ámbito de cinco horas de viaje desde Sincelejo queda incluida una población de casi 7,5 millones de habitantes, la mayor concentración demográfica en ese radio de cualquiera de las ciudades del Diamante (FINDETER, 2014, p. 244).

Con base en lo anterior, Sincelejo estaría cumpliendo con los elementos que identifica la TLC. Su dificultad radica en que aún no alcanza la interacción que posibilita la conectividad interna y externa con su entorno inmediato, lo que permitiría a Sincelejo, como ciudad central, concentrar la oferta de bienes y servicios en el desarrollo de infraestructura para la movilidad vial y logística.

Debido al protagonismo que adquieren las ciudades en el marco del TLC, se ha pedido a los gobiernos locales que tomen en cuenta en sus Planes de Ordenamiento Territorial la forma de interacción interna con su región, la nación y el resto del mundo. En este sentido, se ha acuñado el término de Territorio Inteligente, el cual engloba un nuevo concepto espacial conocido como Ciudad-Región, el cual "supera los límites geográficos y administrativos tradicionales, equiparándose al concepto de territorio funcional" (Calderero, Pérez, \& Ugalde, 2006, p.40). Entonces, ¿cuáles son los parámetros que definen la inteligencia de un territorio? Se argumenta que la competitividad y sostenibilidad mide la inteligencia a través de un modelo teórico que ayuda a construirlo, 
basado en el estudio de tres dimensiones: Económica, Social y Físico-Construida, siendo la arquitectura general del modelo la siguiente:

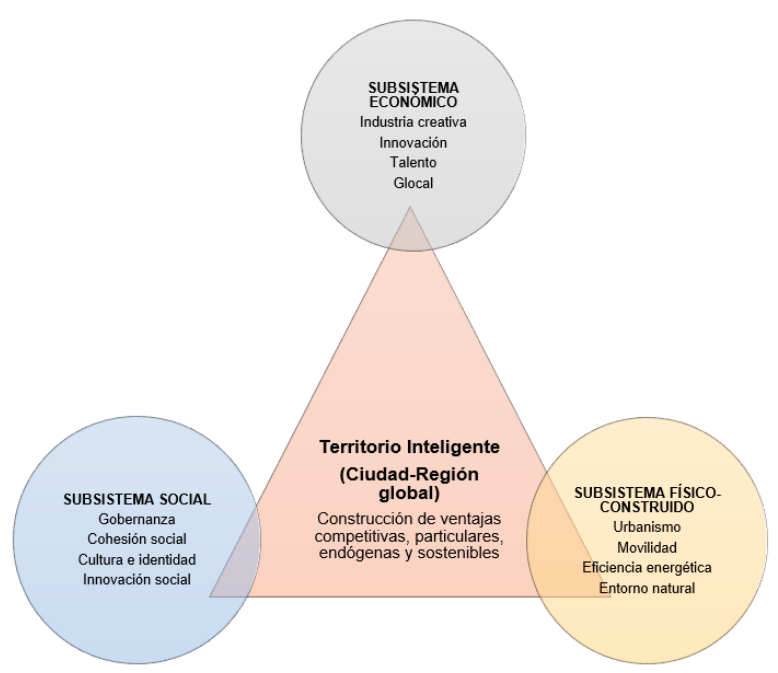

Figura 1. Arquitectura general del modelo. Fuente: Esteban, Ugalde, Rodríguez, \& Altuzarra, 2008, p. 7.

Se trata del modelo conceptual conocido como JakinBask: Territorio Inteligente formulado entre la Fundación Labein y tres facultades de la Universidad del País Vasco (Calderero, Pérez, \& Ugalde, 2006), del cual se puede decir que la incursión de estos modelos en las políticas de los países, entre ellos Colombia, da respuesta a los modelos estáticos y al peso que los desarrollos tecnológicos que imponen la teoría económica, dado lugar a la idea del desarrollo endógeno local y regional, desde presupuestos que estiman que los territorios poseen potencialidades diferenciadoras que pueden desarrollar. De acuerdo con la Figura 1 , el territorio representa el corazón del sistema, es el eje vertebrador del desarrollo sostenible desde las perspectivas económica, social y físico-construida, configurando los subsistemas que poseen unos componentes, los cuales se definen a continuación a fin de denotar su importancia en términos de la competitividad y sostenibilidad parámetros que, según el modelo, mide la inteligencia de un territorio:
En el subsistema económico se desarrolla los siguientes componentes: Industria creativa, se entiende en un Territorio Inteligente como industria del conocimiento, considerada estratégica porque debe promover la Investigación, Desarrollo Tecnológico e Innovación en el ámbito local y lograr crear industrias asentadas en el territorio $y$, por tanto, generadoras de empleos y bienestar para sus ciudadanos.

El componente de Innovación es la importancia de la base de fabricación nacional robusta que se expresa en tres contextos: Empleo, Seguridad Económica y Nacional, e Innovación. La Innovación es el factor crítico del crecimiento. Los países que establecen vínculos poderosos entre la Investigación en el laboratorio y la nueva fabricación están emergiendo como los que más se benefician de sus capacidades innovadoras. (Schmidt, 2013).

El Talento concibe que el motor de desarrollo son las personas y no las empresas. Para Richard Florida (2012), una ciudad o un país atraen a la 'clase creativa' porque poseen un clima social que posibilita que sus habitantes puedan desarrollar todo su potencial. De esta manera entra a la ecuación de las 3T: Talento, Tecnología y Tolerancia, una nueva, el Territorio, en el que la clase creativa establece un modo de vida alternativo" (Alonso, 2011, p.28). Finalmente, el componente Glocal, se refiere a la capacidad que tiene un territorio para deslocalizarse desde su ámbito local, antes físico, para insertarse al mundo, ahora virtual, configurándose georelaciones posibilitadas por las Tecnologías de la Información y comunicación.

Ahora bien, para los componentes integrantes de cada subsistema, se identifican unos vectores, que son las áreas más estratégicas para mejorar el nivel de vida de un territorio. El desarrollo de estas áreas se logra de forma expedita en la medida en que se dispone de factores de éxito, que el modelo denomina catalizadores. En la Tabla 1 se relacionan los que corresponden al subsistema económico: 
Buelvas-Salgado - Lineamientos de ordenamiento y desarrollo territorial desde el modelo de territorios inteligentes

Tabla 1. Catalizadores del subsistema económico.

\begin{tabular}{|c|c|c|c|c|}
\hline \multirow{2}{*}{ Vectores } & \multicolumn{4}{|c|}{ Catalizadores } \\
\hline & Valores & Agente principal & Infraestructura & Tecnología \\
\hline $\begin{array}{l}\text { Industria creativa } \\
\text { (bienes y servicios) }\end{array}$ & Emprendimiento & Sector productivo & Simbiosis industrial & $\begin{array}{c}\text { Eco-eficiencia, TIC, } \\
\text { biotecnologías, } \\
\text { nanotecnología }\end{array}$ \\
\hline $\begin{array}{c}\text { Innovación } \\
\text { (Conocimiento y } \\
\text { tecnología) }\end{array}$ & $\begin{array}{l}\text { Conocimiento, } \\
\text { innovación }\end{array}$ & $\begin{array}{l}\text { Sistema ciencia, } \\
\text { tecnología e } \\
\text { innovación }\end{array}$ & $\begin{array}{l}\text { Parques científicos, } \\
\text { eco-parks, parques } \\
\text { urbanos, laboratorios. }\end{array}$ & $\begin{array}{l}\text { Eco-innovación } \\
\text { e-innovación. }\end{array}$ \\
\hline Talento (RRHH) & Talento, creatividad & $\begin{array}{l}\text { Sistema educativo y } \\
\text { formación }\end{array}$ & Universidad, campus & $\begin{array}{l}\text { e-learning, contenidos } \\
\text { inteligentes, web } 3.0\end{array}$ \\
\hline Glocal (Capital) & $\begin{array}{l}\text { Internacionalización } \\
\text { Identidad }\end{array}$ & Sector empresarial & Parques empresariales & $\begin{array}{c}\text { e-business, empresas } \\
\text { en red, ecosistemas } \\
\text { digitales }\end{array}$ \\
\hline
\end{tabular}

Fuente: Esteban, Ugalde, Rodríguez, \& Altuzarra, 2008.

Continuando con el subsistema físicoconstruido, el cual se constituye inicialmente por el vector del Urbanismo, quien se redimensiona en materia de diseño o regeneración de espacios, observando los parámetros de sostenibilidad y funcionalidad. Se ocupa, por tanto, de la mezcla de uso orientada a acercar trabajo, vivienda y ocio, los cuales adapta a la estructura social y demográfica; concibe el patrimonio en su valía sociocultural al igual que el suelo; respeta las áreas vírgenes, la regeneración de espacios urbanos y suburbanos degradados; mantiene un especial interés por el fenómeno del urbanismo difuso "sprawl" y se ocupa del medio rural al concebir redes de comunicación física entre la ciudad con su región.

El siguiente componente es el de Movilidad. Se relaciona con el sistema de transporte de un territorio, su uso, y el diseño urbano; la construcción de infraestructura de comunicación físicas y digitales, observa la calidad y el impacto socio-económico y medioambiental, siendo clave la oferta multimodal, buscando minimizar el número de viajes necesarios para que los ciudadanos desempeñen sus funciones habituales en el territorio, haciéndose más efectivos los flujos y la conectividad de mercancías y de ciudadanos tanto a nivel interno como hacia el exterior.
La Eficiencia Energética es otro componente que se enfoca en el uso de las Tecnologías Renovables y Limpias como fotovoltaicas, termo solar, eólica y de cogeneración. El diseño urbano y arquitectónico del Territorio Inteligente debe orientarse a la arquitectura bioclimática. Igualmente, a los soportes informáticos flexibles siendo claves para la creciente y compleja gestión energética.

Por último, el componente de Medio Ambiente radica en que el territorio se preocupa de manera especial por los recursos básicos tales como el agua y el aire. En el caso del agua, el territorio promueve el aprovechamiento de las aguas pluviales, depuración de aguas grises y negras, mantenimiento de las capas freáticas, creando pavimentos permeables y cultivando espacios vegetales. También promueve políticas de reciclado de desechables tales como aceites y otros residuos grasos para aprovecharlos como biocombustibles. El sector de la construcción en el Territorio Inteligente racionaliza el uso de materiales y desechos. Se fomenta la concienciación social en los temas medioambientales como elemento clave. En la Tabla 2 se relacionan los que corresponden al subsistema físico-construido: 
Tabla 2. Catalizadores del subsistema físico-construido.

\begin{tabular}{|c|c|c|c|c|}
\hline \multirow{2}{*}{ Vectores } & \multicolumn{4}{|c|}{ Catalizadores } \\
\hline & Valores & Agente principal & Infraestructura & Tecnología \\
\hline $\begin{array}{c}\text { Urbanismo } \\
\text { (diseño urbano) }\end{array}$ & $\begin{array}{c}\text { Diversidad, integración } \\
\text { y ahorro }\end{array}$ & $\begin{array}{l}\text { Construcción, } \\
\text { materiales }\end{array}$ & Vivienda & $\begin{array}{c}\text { Biomateriales, } \\
\text { domótica, GIS, } \\
\text { bioconstrucción, IFC. }\end{array}$ \\
\hline $\begin{array}{c}\text { Movilidad } \\
\text { (infraestructura) }\end{array}$ & $\begin{array}{c}\text { Ahorro, transporte } \\
\text { público }\end{array}$ & Transporte & $\begin{array}{l}\text { Redes de transporte, } \\
\text { logística }\end{array}$ & $\begin{array}{c}\text { Ubicuidad, inteligencia } \\
\text { ambiental }\end{array}$ \\
\hline $\begin{array}{l}\text { Eficiencia } \\
\text { energética } \\
\text { (energía) }\end{array}$ & $\begin{array}{l}\text { Ahorro, eficiencia, } \\
\text { energías limpias }\end{array}$ & Energía & $\begin{array}{c}\text { Micro redes, redes de } \\
\text { BT }\end{array}$ & $\begin{array}{l}\text { Energías renovables, } \\
\text { nuevos combustibles, } \\
\text { BIPV, PCM, aislamiento }\end{array}$ \\
\hline $\begin{array}{l}\text { Medio Ambiente } \\
\text { (aire, agua, } \\
\text { desecho }\end{array}$ & Medio natural & $\begin{array}{c}\text { Medio Ambiente, } \\
\text { urbanismo }\end{array}$ & $\begin{array}{c}\text { Abastecimiento, } \\
\text { saneamiento, } \\
\text { depuración, redes } \\
\text { neumáticas, RCD, RSU. }\end{array}$ & $\begin{array}{l}\text { Permacultura, } \\
\text { ECIA, sinergia } \\
\text { de subproductos, } \\
\text { desalinización, } \\
\text { digestores. }\end{array}$ \\
\hline
\end{tabular}

Fuente: Esteban, Ugalde, Rodríguez, \& Altuzarra, 2008

Finalmente, el subsistema social en el que se desarrollan los componentes de la Gobernanza, el cual asemeja el territorio a una empresa, construyéndose a largo plazo y recurre al debate frente a los retos y las estrategias, introduce la gestión y planificación, el marketing, la financiación público-privados. Logra integrar todas las dependencias para orientarlas a la competitividad del territorio; y recurre a la descentralización para deslocalizarse y promocionar sus ventajas al tiempo que promueve la inversión extranjera directa. Se vale de las Tecnologías de la Información para difundir sus mejores prácticas de gobierno y gestionar el territorio desde la comunicación permanente con los ciudadanos para la toma de decisiones. Seguidamente, el componente de Cohesión Social, que se entiende en un Territorio Inteligente como su responsabilidad de centrarse y en asegurar el bienestar de sus ciudadanos sin exclusión social por razones de discapacidad, migración, edad u otras características diferenciales, otorgando a todos, acceso a los servicios sociales. De esta manera se asegura altos niveles de cohesión social, al tiempo que se retiene un talento humano que, al identificarse con el territorio, lo defiende.
Asimismo, la Cultura e Identidad la evidencia un Territorio Inteligente relacionando el desarrollo de los territorios con la cultura y los valores, específicamente el respeto al medio ambiente, tolerancia, talento, conocimiento, innovación, cooperación, emprendimiento, el bien común y la salud o la calidad. Por otra parte, la Innovación social persigue la búsqueda de soluciones creativas e innovadoras a problemas sociales, mediante el fomento y desarrollo del emprendimiento social y de ONG, con el fin de aumentar la eficiencia en los servicios y la cohesión de la comunidad. Algunos ejemplos de innovación social de amplio impacto se refieren a la idea de responsabilidad social, la Universidad Abierta, el Comercio Justo, Greenpeace, Amnistía Internacional y el software Linux. En la Tabla 3 se relacionan los que corresponden al subsistema social.

Aunque el modelo puede ser aplicado al territorio que abarca una región, ciudad, área metropolitana o barrio, en esta Investigación se empleó la acepción de territorio en relación con la idea de CiudadRegión. 
Tabla 3. Catalizadores del subsistema social.

\begin{tabular}{|c|c|c|c|c|}
\hline \multirow{2}{*}{ Vectores } & \multicolumn{4}{|c|}{ Catalizadores } \\
\hline & Valores & Agente principal & Infraestructura & Tecnología \\
\hline $\begin{array}{l}\text { Gobernanza } \\
\text { (políticas) }\end{array}$ & $\begin{array}{l}\text { Corresponsabilidad, } \\
\text { eficiencia }\end{array}$ & $\begin{array}{l}\text { Administración } \\
\text { (políticas) }\end{array}$ & & $\begin{array}{l}\text { e-gobierno, web } \\
\text { 2.0, e-participación, } \\
\text { realidad virtual }\end{array}$ \\
\hline $\begin{array}{l}\text { Cohesión social } \\
\text { (servicios sociales) }\end{array}$ & $\begin{array}{c}\text { Integración, equidad, } \\
\text { conciliación }\end{array}$ & $\begin{array}{l}\text { Sectores público y } \\
\text { privado y sociedad civil }\end{array}$ & $\begin{array}{l}\text { Equipamientos } \\
\text { sociales }\end{array}$ & $\begin{array}{c}\text { e-inclusión, domótica, } \\
\text { e-salud }\end{array}$ \\
\hline $\begin{array}{c}\text { Cultura e identidad } \\
\text { (valores y } \\
\text { comunicación) }\end{array}$ & $\begin{array}{l}\text { Multiculturalidad, } \\
\text { identidad, tolerancia }\end{array}$ & Cultura y comunidad & $\begin{array}{c}\text { Museos temáticos, arte } \\
\text { electrónico, nuevos } \\
\text { espacios }\end{array}$ & $\begin{array}{c}\text { Web } 2.0, \text { contenidos } \\
\text { inteligentes, realidad } \\
\text { virtual }\end{array}$ \\
\hline $\begin{array}{l}\text { Innovación social } \\
\text { (cambio social) }\end{array}$ & $\begin{array}{l}\text { Innovación, bienestar, } \\
\text { corresponsabilidad }\end{array}$ & Tercer sector & Redes de comunicación & e-innovación, web 2. \\
\hline
\end{tabular}

Fuente: Esteban, Ugalde, Rodríguez, \& Altuzarra, 2008.

\section{METODOLOGÍA}

La Investigación sigue la metodología de Investigación estratégica de JakinBask: territorios inteligentes, expuesto por Esteban, Ugalde, Rodríguez, \& Altuzarra (2008), el cual está orientado a la generación de un nuevo marco teórico y metodológico que ayuda a la comprensión del desarrollo territorial. En esta dirección, el análisis sobre Sincelejo como Territorio Inteligente pretendió identificar los factores que, presentes en la teoría permite caracterizar y explicar a Sincelejo en sus subsistemas económico, social y físico-construido para visionar brechas a cerrar, para lo cual se consultó fuentes de datos extraídos de documentos institucionales, estudios sectoriales, buscadores de Internet.

Se obtuvo, igualmente, información primaria proveniente de entrevistas en profundidad a personas expertas en ordenamiento y desarrollo territorial conocedoras y planificadoras del contexto municipal de Sincelejo, en particular de la Oficina de Planeación Municipal, como participantes directamente implicados en la toma de decisiones sobre ordenación y desarrollo de Sincelejo.
La información analizada se estructuró en los subsistemas del Modelo de Territorio Inteligente: Subsistemas Económico, Subsistemas Físico-Construido y Subsistemas Social, a fin de establecer una visión holística de Sincelejo frente al concepto de Territorio Inteligente y las variables que lo condicionan, organizada la información, fue posible discutir a la luz de la teoría relacionada en el marco teórico, los lineamientos para hacer de Sincelejo un Territorio Inteligente.

\section{RESULTADOS Y DISCUSIÓN}

Sincelejo configura una Ciudad-Región que, en su acepción formal, es el espacio de estructura policéntrica que define un patrón espacial central respecto a un área de influencia ampliado con el que crea intercambios sociales, culturales y comerciales. En la Imagen 2 se evidencia como la ciudad de Sincelejo se interrelaciona con los municipios cercanos $y$, de igual forma, con los corregimientos próximos. 

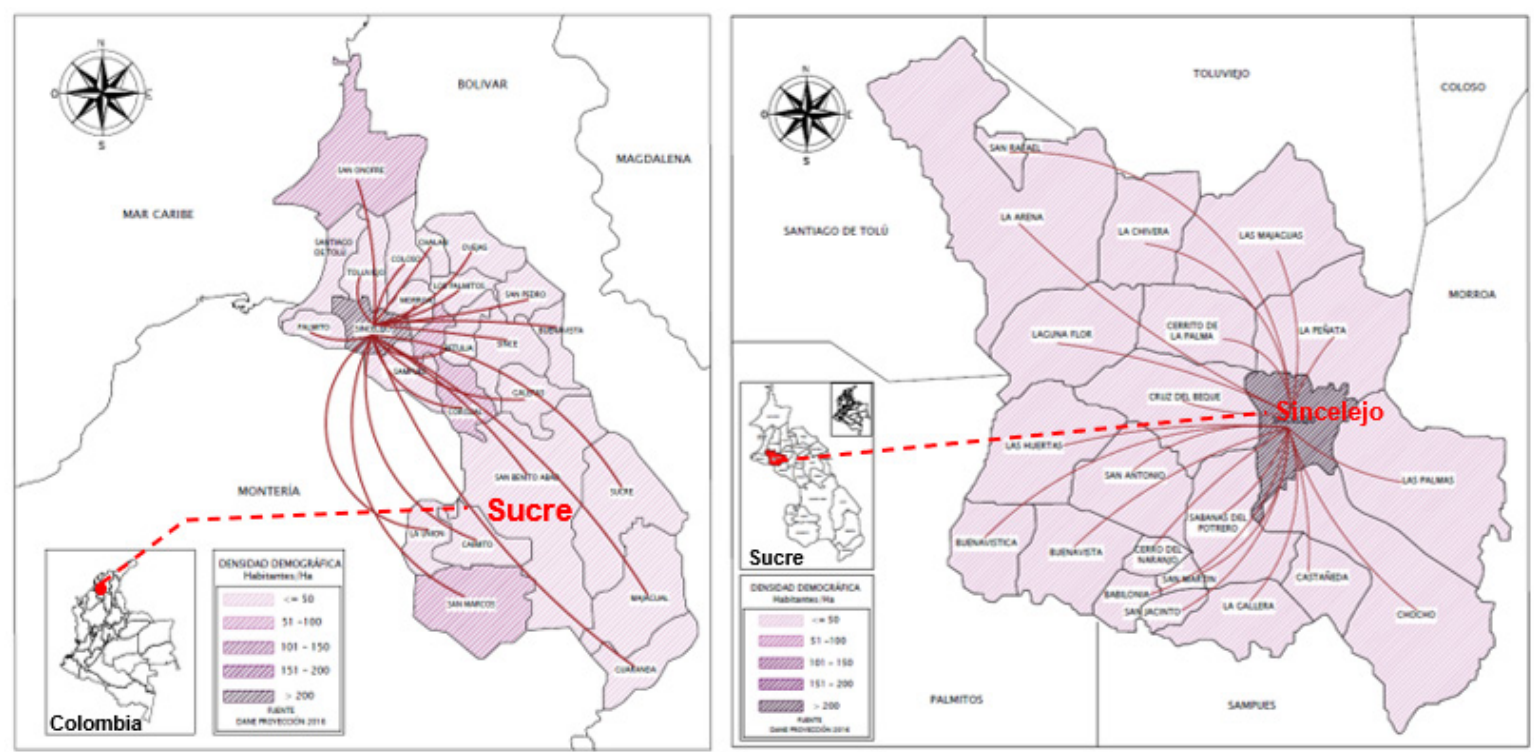

Figura 2 Sincelejo Ciudad-Región desde la perspectiva del departamento de Sucre, Colombia.

Elaboración propia según (DANE, 2016).

Sincelejo, en el subsistema económico se caracteriza en el componente de Industrial Creativa por ser poco diversificada y se limita principalmente a la fabricación de cementos $(87,1 \%)$, seguida de la producción de alimentos $(0,7 \%)$. Presenta escasa participación de los bienes agropecuarios para la alimentación, los cuales se venden en los mercados internos y externos sin ningún valor agregado (Cámara de Comercio de Sincelejo, 2017, p. 23). De acuerdo con él (DANE, 2014), de los 116 mil ocupados en Sincelejo, la industria emplea a 11.000 personas, lo que identifica a un sector industrial incipiente, concentrándose la ocupación laboral en los sectores de comercio y servicios. Asimismo, el $99.49 \%$ del parque empresarial de Sincelejo está compuesto por la Micro, Pequeña y Mediana Empresa, siendo solo 7 las empresas grandes ubicadas en el municipio.

La Ciencia y Tecnología -como indicador global de competitividad- ubica a Sincelejo en el puesto 19 entre 22 ciudades colombianas, en especial entre las ciudades capitales del Caribe colombiano para el año 2014 (CEDEC, 2015, p. 20). Esto respecto al vector de Innovación.

Según el componente de Talento, Sincelejo ha alcanzado una muy buena cobertura en cuanto a Educación Media y Secundaria cubriendo al $100 \%$ de las personas que acuden a esa formación, pero exhibe problema en cuanto a la Educación Superior la cual presenta baja cobertura (27\%) (Plan de Desarrollo de Sincelejo, 2016 - 2019, p. 145).

Respecto a lo Glocal, son incipientes las iniciativas virtuales que desarrolla Sincelejo, pero desde el 2014 cuenta con un Centro Vivelab a partir de la alianza entre el MinTIC, Colciencias, Gobernación de Sucre, Parquesoft Sucre y Cámara de Comercio de Sincelejo. Está dotado para desarrollar contenidos digitales, cuenta con 7 laboratorios para talleres formativos; Centro de Entrenamiento; Sala de Desarrollo de Aplicaciones Móviles; Laboratorio de Contenido Digital; Laboratorio de Producción y Postproducción de Vídeo Digital; Laboratorio de Captura de Movimiento; Laboratorio de Audio y Sonido, entre otros espacios. (Quiroz, 2014).

Sincelejo, en el subsistema físico-construido, en el componente de Urbanismo tiene un área urbana de $292 \mathrm{Km} 2$ habitado por 270.031 personas para el año 2016. De acuerdo con el (INCODER, 2017, p.7), "el municipio de Sincelejo presenta densidad relativamente muy alta, con promedio de 
925 habitantes por $\mathrm{Km} 2 "$, lo que indica que frente al ritmo de crecimiento de la población de 1.49 interanual, es relativo poco espacio, al mismo tiempo que se registra una baja densidad de unidades de vivienda - de 30 a 35 viviendas/ha - que conlleva a que la ciudad presente limitantes al crecimiento físico, presentándose el fenómeno Sprawl.

Respecto a la Movilidad, Sincelejo cuenta con una red vial urbana de aproximadamente 512 kilómetros de longitud; obedece a un esquema de tipo radial en donde la gran mayoría de los corredores actúan como ejes arteriales que comunican el centro de la ciudad con las zonas residenciales ubicadas en la periferia de Sincelejo (Plan de Desarrollo de Sincelejo, 2016 - 2019, p.191). En Sincelejo, Metro Sabanas S.A.S fue creada para implementar y construir el Sistema Estratégico de Transporte Público (SETP), el cual entró en funcionamiento el 8 de agosto de 2015 (Metro Sabanas, 2015, p. 21). Esta misma entidad, dentro de sus proyecciones, propone un plan de movilidad que va desde el arreglo de vías, señalización complementaria a la existente, un terminal de transporte intermunicipal de integración regional; la construcción de ciclovías y zonas de parqueo vehicular en zonas estratégicas de la ciudad. El actual SITP dentro de las soluciones al transporte masivo, apostó por introducir 85 busetas de escasa capacidad de carga por recorrido; 23 solo tienen capacidad para 6 pasajeros; y las otras 27 tienen capacidad para 25 personas.

En el componente de Eficiencia Energética, el $26 \%$ de las emisiones globales de Gases Efecto Invernadero (GEI) se generan por la demanda de energía. Las ciudades consumen el $75 \%$ de la energía y producen más del $80 \%$ de las emisiones de CO2 y otros GEI que se generan anualmente en el planeta (Universidad Externado de Colombia, 2013, p.34). Sincelejo sustenta su eficiencia energética en los sistemas de generación del país, para el 2013 provenientes en un 67\% de hidroeléctricas y un $33 \%$ de generación térmica y $0.1 \%$ eólica.

Por último, en este subsistema del componente de Medio Ambiente, Sincelejo enfrenta la degradación de 27.900 hectáreas por erosión hídrica; 5.913,51 hectáreas sometidas a quemas (CARSUCRE, 2012, p.59), deficiencia en disposición de residuos sólidos; dependencia del agua a partir del acuífero de Morroa, fuente subterránea de abastecimiento de agua potable para una población de aproximadamente 500.000 habitantes de las zonas urbanas y rurales de municipios conexos a Sincelejo. A Sincelejo, el agua para consumo humano llega tres o cuatro días de la semana; el sistema de acueducto data de hace veinte años y fue proyectado para 100.000 habitantes, hoy Sincelejo tiene una población cercana a los 300.000 habitantes y el $40 \%$ de los barrios son subnormales (Cámara de Comercio de Sincelejo, 2017, p.28).

En continuidad con el subsistema social, se analiza el componente de la Gobernanza desde los indicadores mundiales de gobernabilidad para Colombia; el municipio de Sincelejo no presenta déficit fiscal, siendo calificada sobresaliente en desempeño fiscal (82.39) por el DNP y ocupa el puesto 37 entre 1.102 municipios. Entre las 32 capitales, Sincelejo ocupa el cuarto puesto. El 90\% de los recursos de renta propias se captan en el sistema financiero con tecnología de código de barras, lo que genera seguridad en el manejo financiero (Alcaldía de Sincelejo, 2015).

Conforme a la Cohesión Social, Sincelejo para el año 2015 el porcentaje de población con Necesidades Básicas Insatisfechas (NBI) resulta de $42,02 \%$ más alto que el promedio nacional $27.78 \%$; la tasa de desempleo fue de $9,8 \%$, siendo la tasa de subempleo de $34,6 \%$. En cuanto a la cobertura de servicios públicos en Sincelejo, se encuentra una cobertura nominal de 82.8 , disponibilidad de 8 horas de servicio y un factor de continuidad de 0.44, para una cobertura real de 36.4; una cobertura nominal de alcantarillado de 81.4 a nivel urbano, a nivel rural no se presta este servicio (CARSUCRE, 2012). Respecto a la forma de ocupar el territorio, existe un déficit cuantitativo de 7.667 viviendas hacía el norte y sur de la ciudad, que son sectores donde se apuestan los estratos 1 y 2 de la población, siendo al mismo tiempo barrios con déficit cualitativo de vías, con menor cobertura del servicio de transporte público 
y muchas veces sin las adaptaciones que requieren las poblaciones funcionalmente diversas.

El patrimonio material e inmaterial, pertenece al componente de Cultura e Identidad; según el POT, se debe inventariar en cuanto a legados arquitectónicos. La ciudad en su Centro Histórico posee una construcción urbana de estilo republicano, representada en 39 inmuebles, los cuales han sido exonerados del pago de impuesto predial como estrategia para asegurar su conservación.

Según el componente de Innovación social, en Sincelejo no se cuenta con iniciativas de una universidad abierta que ofrezca cursos masivos en línea; tampoco se cuenta con experiencias en comercio justo, o el empleo u oferta de software abierto. En otros indicadores como Responsabilidad Social Empresarial (RSE) y medios electrónicos para interactuar con el gobierno local presenta mejor desempeño.

Un estudio realizado en el 2014 reporta que las actividades de responsabilidad social empresarial entre las MiPymes de Sincelejo están orientadas internamente y con muy poca relación con la gestión de sus actividades empresariales (León, Castán, \& Afcha, 2014). Esta situación mejora en la medida que el tamaño de la empresa aumenta, haciéndose más evidente la adopción de programas de RSE enfocados a la razón social de la empresa.

Desde un marco legal, Sincelejo CiudadRegión, está concebida dentro del enfoque territorial como centro funcional y jerárquico que sigue los lineamientos de la Ley 1753 de 2015, y por tanto orienta su planificación como centro urbano de intercambios con los territorios vecinos, hasta que llegue a configurar un área metropolitana que le permita beneficiarse de las economías de aglomeración, tal como lo logran en el país las ciudades de Bogotá, Medellín y Cali, tríade conocido como el triángulo de oro, en cuanto generan escalamiento económico que les permite la más alta contribución al PIB (76\%) de la nación (UNAL, 2014). En ese orden de ideas se plantea que "el Departamento, de manera conjunta con la ciudad capital deben definir los equipamientos que esta requiere, como también el ordenamiento de su territorio para que pueda cumplir de manera eficiente y oportuna con su condición de Ciudad Región" (Plan de Desarrollo de Sincelejo, 2016 - 2019, p.32).

Las economías de escala determinan unos patrones de intercambio optimizados, desde los que se reducen los costos medios de la producción en un largo plazo, efectos prácticos susceptibles de operarse para generar desarrollo socioeconómico y mejoramiento de la calidad de vida de quienes habitan un territorio. Tal comprensión se encuentra inscripta en el Plan Nacional de Desarrollo (PND) 2014-2018 'Todos por un nuevo país', en el que se propone el fortalecimiento del Sistema de Ciudades como motor del crecimiento de la nación, determinándose en "la Visión Colombia 2019: Construir Ciudades Amables, por tanto se orienta la Política Nacional al propósito de Consolidar el Sistema de Ciudades de Colombia a 2035 (Plan Nacional de Desarrollo, 2014-2018, p.27).

Desde la inscripción de Sincelejo en el Diamante Caribe como Territorio Inteligente, se engloba un nuevo concepto espacial, bajo el término de Ciudad-Región en los siguientes términos: superar la noción de ciudad aislada, entendiendo el espacio regional como una estructura policéntrica compuesta por la suma de ciudades y su propio entorno natural. Por otro lado, el concepto de Territorio Inteligente se iguala al concepto de territorio funcional 0 , lo que es lo mismo, al concepto de territorio basado en áreas naturales de intercambio económico-cultural, superando la definición tradicional del territorio en términos políticoadministrativos.

Sincelejo y el Departamento, exportan el grueso de la producción primaria o capital natural en el que posee ventajas comparativas (bovinos en pie, cemento legumbres y tabaco); de acuerdo con Proextport, de US\$23 millones fue el total de las exportaciones de Sucre en 2013, correspondiendo el 98,28\% a exportaciones no mineras iguales o mayores a US $\$ 10.000$, 
realizadas por 19 empresas (Proexport Colombia, 2013, pág. 3).

Con referencia al talento, presenta dificultades para la formación de capital intelectual de alta cualificación para el territorio, donde componentes como la variedad de personas formadas también tiene relación directa con la oferta formativa de las universidades, concentrándose la oferta académica en las siguientes áreas:

En Ciencias Sociales: Administración con un $18,75 \%$, Educación $14,01 \%$, Contaduría Pública 11,05\%, Derecho 10,22\% y Psicología 7,07\%.

En Ingenierías $17,41 \%$, siendo la Ingeniería de Sistemas con más estudiantes 5,95\%, seguido de la Civil con $3,26 \%$ e Industrial con $2,39 \%$.

En Agroindustria y afines se destacan la Ingeniería Agrícola y Forestal con 1,57\% e Ingeniería Agroindustrial y Alimentos $1,57 \%$, Zootecnia 1,86\%, Biología 2,52\% y Agronomía 0,44\% (Red Nacional de Agencias de Desarrollo Local, 2013) (SNIES, 2016).

Es decir, existe una oferta de formación orientada a las disciplinas tradicionales, sin orientación a los campos que concentran la $\mathrm{I}+\mathrm{D}+\mathrm{i}$. Por su parte, la construcción como catalizador principal del urbanismo muestra evolución en la ciudad. En 2015, en Sincelejo, registró un crecimiento de 4,3\% en el número de licencias aprobadas respecto el año anterior, al pasar de 253 licencias en 2014 a 264 en 2015. De este total, 221 fueron destinadas a vivienda, esto es 3,8\% más que el número de licencias aprobadas para este destino en 2014 (con 213). La participación de licencias para vivienda en el total ascendió a $83,7 \%$ en 2015 . En términos de área a construir, se aprobaron 181.009 m2 en 2015 para Sincelejo; es decir, disminuyó $6,7 \%$ respecto a 2014, en el que se aprobaron $194.104 \mathrm{~m} 2$. La vivienda tuvo una participación de $79,1 \%$ en el área a construir en 2015 (correspondiente $143.224 \mathrm{~m} 2$ ) y una variación negativa de $10,3 \%$, respecto al año anterior (ICER DANE, 2015, p.46).

\section{CONCLUSIONES}

Sincelejo, requiere que sus líderes, representados en la clase dirigente, comprendan que muchas de las soluciones a sus propios problemas está en sus propias capacidades de tipo natural (agro - cultura - turismo) y en su gente (creativa) para que con base en información y oportunidad trace la visión de un territorio que está por construir, pues se trata de una ciudad intermedia, cuya etapa aún embrionaria puede apropiarse del conocimiento del mundo para adaptarlo y apropiarlo en su propio desarrollo.

La noción de Territorio Inteligente es una consecuencia de estar incursos en la sociedad del conocimiento para adecuar los procesos de desarrollo por los que transita la humanidad. Ciudades intermedias, como Sincelejo, están llamadas a estudiar las tendencias a su alrededor. Existen múltiples casos de ciudades de igual o menor tamaño desarrollándose desde estos preceptos, existe la legislación para tomar decisiones dentro del marco de la ley para deslocalizar el territorio, y también se cuenta con las estrategias de financiación que permitirían emprender un plan a largo plazo.

Sincelejo ha hecho parte de estudios recientes del Banco Interamericano de Desarrollo y se nombra también en el Plan Nacional de Desarrollo 2014-2018 "todos por un nuevo país" como una ciudad nodal; igualmente es de interés de Findeter como ciudad intermedia y está dentro de la focalización del proyecto Diamante Caribe. El atractivo de estos tamaños de población conlleva la idea de desconcentrar las grandes ciudades, existe un interés por explorar nuevos territorios que puedan crecer de manera planificada, tratando de no repetir en su planeación las externalidades negativas que viven las ciudades grandes del país por cuenta de la polución, el crecimiento desbordado, las grandes distancias que deben recorrerse para llegar a los puntos de trabajo. La tendencia es a concentrar trabajo, vivienda y ocio para hacer del territorio un mejor hábitat. 
Con la mejor de las intenciones y reconociendo que Sincelejo es un territorio con capacidad de aprender, debe decirse que el reciente sistema de transporte público que introdujo 85 busetas, algunas de solo 6 pasajeros, se aleja del concepto de transporte masivo, lo que genera deficiencia en términos de ahorros económicos y sostenibilidad ambiental, pues se trata de vehículos a base de combustibles fósiles, en esta ocasión gasolina, que contaminan desde la emisión de Gases Efecto Invernadero (GEI). Si a esto se suman 42.000 mototaxis que diariamente ofrecen servicios de transporte en la ciudad, se genera una situación ambiental crítica, aún sin considerar el parque de taxis públicos y transporte privado. Sincelejo debe preguntarse qué está pasando con los residuos de aceite de este enorme parque automotor al momento de ser lavados. La disposición de estos desechos ¿podría contaminar fuentes hídricas como el acuífero de Morroa, única fuente de agua potable del municipio? Urgen soluciones como el rediseño vial a partir de supermanzanas, uso de vehículos de transporte masivo, intermodalidad, control central del transporte público, formación de públicos.

El mototaxismo ha logrado 'democratizar el transporte público de Sincelejo', he ahí su enorme éxito, además de otros factores como las escasas fuentes de empleo. Una solución a partir de estimar cifras económicas: 42.000 motocicletas dejan una utilidad a cada mototaxista de $\$ 10.000$ diarios netos, lo que equivale a una transacción diaria neta de $\$ 420.000 .000$ pesos. La propuesta es que los mototaxis sean agremiados en una empresa público-privada. Ellos entregarían la motocicleta como capital para la compra de buses articulados de mayor tamaño a base de combustible no contaminantes - eléctricos, las rutas se adecuarían a partir del concepto de supermanzanas. Los mototaxistas no volverán a hacer uso de sus motocicletas: recibirán formación empresarial, lo que requiere pedagogía equivalente a su nivel de estudios. Ellos, a cambio, recibirían mensualmente el acumulado de un equivalente a sus ingresos diarios. Además, se harían convenios públicoprivados para controlar la venta de motos con destino a ser mototaxi. Se vincularía a la cooperación internacional en la propuesta, la cual debe elaborarse a partir de las ideas aquí expuestas.

Otros aspectos puntuales para mejorar, a partir de lo que deja apreciar el presente estudio estarían determinados por:

Ampliar cobertura de las TIC a la totalidad de la población del Municipio de Sincelejo.

Crear una Oficina de Relaciones Internacionales al interior del gobierno municipal.

Deslocalizar a Sincelejo para integrarlo a las regiones del mundo.

\section{REFERENCIAS}

Alcaldía de Sincelejo. (2015). Informe de rendición de cuentas año 2014 Alcaldía de Sincelejo, Plan de Desarrollo Un Alto Compromiso. Obtenido de http://sincelejo-sucre. gov.co/apc-aa-files/39663166316363343139313033356162/informe-de-rendicion-decuentas-2014.pdf

Alonso, Á. (2011). Productividad, competitividad y salarios en ciudades grandes: La clase creativa. Encrucijadas, Revista Crítica de Ciencias Sociales, No 2, p. 23-33. Disponible en: $\underline{\text { https://dialnet.unirioja.es/servlet/articulo?codigo }=3819519}$

BID. (2015). Las ciudades intermedias con mayor potencial en Colombia: Un sistema de identificación. Obtenido de Patricia Torres Arzayús; Carlos Jorge Caice Cuervo: https:// publications.iadb.org/bitstream/handle/11319/6890/Las ciudades intermedia 
Calderero, A., Pérez, J., \& Ugalde, I. (2006). Territorio inteligente y espacios de economía creativa: una primera aproximación conceptual y práctica de Investigación. Economía. Vol. LXXXI-6: 40-44 DYNA Julio-agosto-septiembre, p. 40 - 44. Disponible en: http:// www.cursobioeconomia.mincyt.gob.ar/wp-content/uploads/2016/04/TERRITORIOSINTELIGENTES.pdf

Cámara de Comercio de Sincelejo. (2017). Informe económico del departamento de Sucre. Sincelejo: Recuperado de: http://ccsincelejo.org/wp-content/uploads/2018/02/InformeEcon\%C3\%B3mico-Sucre-2017.pdf

CARSUCRE. (2012). Plan de acción 2012 - 2015. Sincelejo: Corporación Autónoma Regional de Sucre. Disponible en: https://carsucre.gov.co/wp-content/uploads/ PLANDECONTINGENCIREIONAL.pdf

CEDEC. (2015). Indicador Global de Competitividad para 22 capitales colombianas. Cartagena de Indias: Centro de Estudios para el Desarrollo y la Competitividad "CEDEC". Disponible en: https://biblioteca.cccartagena.org.co/xmlui/bitstream/handle/123456789/190/ IGC\%202014.pdf? sequence $=1$ \&isAllowed $=y$

Christaller, W. (1966). Central places in Southern Germany. Fischer (alemán de 1935). Disponible en: https://pdfcookie.com/documents/christaller-central-places-in-southerngermany-1966-o2npeg1363v4

Contraloría Municipal de Sincelejo. (2016). Primer informe ambiental: Estado de los recursos naturales y del ambiente del municipio de Sincelejo. Sincelejo: Recuperado de: https:// contraloriasincelejo.gov.co/wp-content/uploads/2017/11/Informe-Ambiental.pdf

DANE. (2014). Informe de coyuntura económica regional. Bogotá: DANE-Banco de la República. Disponible en: https://repositorio.banrep.gov.co/bitstream/handle/20.500.12134/8759/ icer sucre 2014.pdf?sequence $=1$ \&isAllowed $=y$

DANE. (2016). Colombia: Encuesta de Desarrollo e Innovación Tecnológica - EDIT Industria 2009-2010. Bogotá.: Obtenido de Departamento Administrativo Nacional de Estadística DANE http://formularios.dane.gov.co/Anda 4 1/index.php/catalog/218

Esteban, M. Ugalde, M., Rodríguez, A., \& Altuzarra, A. (2008). Territorios inteligentes: Dimensiones y experiencias internacionales. España: Editorial Gesbiblo S.L. Disponible en: https://books.google.com.co/books?id=ffhnWBn7LuUC\&pg=PT64\&hl=es\&source=gbs selected pages\&cad $=3 \# \mathrm{v}=$ onepage $\& \mathrm{q} \& \mathrm{f}=$ false

FINDETER. (2014). Tomo I. El territorio: Punto de partida para un nuevo desarrollo. Bogotá: Fundación Metrópoli - Microsoft - Financiera del Desarrollo Territorial (FINDETER). Recuperado de: https://issuu.com/findetersa/docs/diamantet1-partida

FLORIDA, R (2012) the rise of the creative class, revisited. Basic Book. Nueva York

García, D. A. (2013). Potencial turístico en la ciudad de Sincelejo (Sucre). Cartagena: Universidad Tecnológica de Bolívar. Disponible en: http://biblioteca.unitecnologica.edu. co/notas/tesis/0064720.pdf

ICER - DANE. (2015). Informe de coyuntura económica regional. Cartagena: Banco de la República. Disponible en: https://www.banrep.gov.co/sites/default/files/publicaciones/ archivos/icer sucre 2015.pdf 
INCODER. (2017). Caracterización sociodemográfica del área de desarrollo rural de Montes de María. Sincelejo: Instituto Colombiano de Desarrollo Rural. Disponible en: https://xdoc. $\underline{\mathrm{mx} / \text { documents/caracterizacion-socio-demografica-de-montes-de-maria-5f481c2713708 }}$

León, G., Castán, J. M., \& Afcha, S. (2014). Responsabilidad social en las Mipymes del municipio de Sincelejo, Sucre. Dimensión Empresarial 13(2), pp. 185-203. Disponible en: http:// ojs.uac.edu.co/index.php/dimension-empresarial/article/view/533/pdf 19

Lösch, A. (1944). Die räumliche ordnung der wirtschaft. Jena, Fischer.

Metro Sabanas. (2 de diciembre de 2015). Formulación del plan de movilidad de Sincelejo y definición de la línea base de transporte público. Obtenido de file:///C:/ Users/FAMILIA\%20TOVAR/Downloads/Informe\%205\%20Etapa\%203\%20Cap10 PlanInstitucionalyMarcoLegal_v6.pdf

Municipio de Sincelejo. (2013). Plan anticorrupción y de atención al ciudadano. Sincelejo: Alcaldía Municipal de Sincelejo. Disponible en: http://cdim.esap.edu.co/BancoMedios/ Documentos\%20PDF/sincelejosucreplananticorrupcionydeatencionalciudadano2013.pdf

Plan de Desarrollo de Sincelejo. (2016 - 2019). Ciudad con visión. Sincelejo: Alcaldía Municipal. Disponible en: https://www.alcaldiadesincelejo.gov.co/Transparencia/ PlaneacionGestionyControl/Plan\%20de\%20Desarrollo\%202016\%20-\%202019.pdf

Plan Nacional de Desarrollo. (2014 - 2018). Todos por un nuevo país. Obtenido de https://colaboracion.dnp.gov.co/cdt/prensa/bases $\% 20$ plan $\% 20$ nacional $\% 20$ de $\% 20$ desarrollo\%202014-2018.pdf

POT. (2015). Decreto N438 de 2015 por medio del cual se adopta el Plan de Ordenamiento Territorial de Sincelejo. Sincelejo: Alcaldía Municipal. Disponible en: https://www. asocapitales.co/nueva/wp-content/uploads/2020/11/Sincelejo Acuerdo147POT 2015.pdf

Proexport Colombia. (2013). Sucre: Oportunidades de negocio para la región en inversión, exportaciones y turismo. Obtenido de http://www.procolombia.co/sites/default/files/ revista de oportunidades_proexport_sucre.pdf

Quiroz, E. (1 de octubre de 2014). Sucre tiene centro ViveLab para el desarrollo de contenidos digitales. El Universal, págs. http://www.eluniversal.com.co/regional/sucre/sucre-tienecentro-vivelab-para-el-desarrollo-de-contenidos-digitales-172719-HUEU267808

Red Nacional de Agencias de Desarrollo Local. (2013). Plan estratégico departamental de ciencia, tecnología e innovación de Sucre: Sucre innova, Sucre se transforma. Obtenido de http://www.colciencias.gov.co/colcienciasdev/sites/default/files/upload/paginas/ pedcti-sucre.pdf

Santacruz, M. (2006). Territorio y desarrollo: camores renovados? Revista de economía y administración de la Universidad Autónoma de Occidente (Alcalá de Henares, España), Vol. 2 (2), 113 - 169. Disponible en: https://revistas.uao.edu.co/ojs/index.php/REYA/ article/view/277

Schmidt, M. (2013). At the hearing before the senate committee on commerce, science and transportation on the role of manufacturing collaborations in a 21 ST century innovation economy. Massachusetts: Massachusetts Institute of Technology. Disponible en: http://web.mit.edu/pie/MIT\%20Martin\%20 Schmidt\%20testimony\%20to\%20Senate\%20Commerce\%20Comm\%2011\%206\%202013.pdf 
Buelvas-Salgado - Lineamientos de ordenamiento y desarrollo territorial desde el modelo de territorios inteligentes

SNIES. (2016). Informes departamentales de educación superior. Bogotá, D.C.: Sistema Nacional de Información de Educación Superior. Disponible en: http://www.mineducacion. gov.co/sistemasdeinformacion/1735/w3-article-212352.html

UNAL. (2014). Triángulo de Oro, corredor clave para economía nacional. Manizales: Universidad Nacional de Colombia. Recuperado de: http://agenciadenoticias.unal.edu.co/detalle/ article/triangulo-de-oro-corredor-clave-para-economia-nacional.html

Universidad Externado de Colombia. (2013). Guía de territorios inteligentes. Bogotá, D.C. : Xpress Estudio Gráfico y Digital S.A. Disponible en: https://issuu.com/observaticsuexternado/ docs/gtci_versi n_2013 2

UPME. (2015). Plan energético nacional Colombia: Ideario energético 2050. Obtenido de Unidad de Planificación Minero Energética (UPME) de Colombia: http://www.upme.gov. co/Docs/PEN/PEN IdearioEnergetico2050.pdf

Vergara, A. (2009). Territorios Inteligentes. Bogotá: Ambiental. Disponible en: http://www. cursobioeconomia.mincyt.gob.ar/wp-content/uploads/2016/04/territorios-inteligentesvergara.pdf

Weber, A. (1929). Theory of the location of industries. Chicago Illinois: Disponible en: http:// www.economia.unam. mx/cedrus/descargas/Libro\%20de\%20Weber.pdf 